\title{
The Evolution of the Scalar Curvature of a Surface to a Prescribed Function
}

\author{
PAUL BAIRD - ALI FARDOUN - RACHID REGBAOUI
}

\begin{abstract}
We investigate the gradient flow associated to the prescribed scalar curvature problem on compact Riemannian surfaces. We prove the global existence and the convergence at infinity of this flow under sufficient conditions on the prescribed function, which we suppose just continuous. In particular, this gives a uniform approach to solve the prescribed scalar curvature problem for general compact surfaces.
\end{abstract}

Mathematics Subject Classification (2000): 58E20.

\section{1. - Introduction}

Let $\left(M, g_{0}\right)$ be a compact Riemannian surface without boundary with scalar curvature $R_{0}=R_{g_{0}}$. A conformal change of the metric $g_{0}$ produces a metric $g=e^{2 u} g_{0}$ having scalar curvature

$$
R=R_{g}=e^{-2 u}\left(-2 \Delta_{0} u+R_{0}\right),
$$

where $\Delta_{0}=\Delta_{g_{0}}$ is the Laplace-Beltrami operator with respect to the metric $g_{0}$.

The prescribed scalar curvature problem is to find conditions on a given function $f: M \rightarrow \mathbb{R}$ in order that it be the scalar curvature of some metric $g$ conformal to $g_{0}$. The corresponding partial differential equation to be solved for $u$, is

$$
f=e^{-2 u}\left(-2 \Delta_{0} u+R_{0}\right) .
$$

Necessary conditions on the function $f$, which are stated in (1.5) below, are needed for the solvability of Problem (1.1). There is an extensive literature concerning sufficient conditions on $f$ which guarantee a solution. For instance, in the negative case: $R_{0} \leq 0$, we refer to Aubin [1]-[3], Bismuth [5] and 
Kazdan-Warner [17]. In the positive case, when $M=S^{2}$; this is the so called Nirenberg problem. It was first solved by Moser [19] for an even function $f$ (in other words when $M=\mathbb{P}_{2}(\mathbb{R})$, condition (1.5)(iii) stated below is necessary and sufficient). Next, Chang-Yang [7] gave a corresponding version of Moser's result for functions satisfying a reflexion symmetry about some plane $f\left(x_{1}, x_{2}, x_{3}\right)=f\left(x_{1}, x_{2},-x_{3}\right)$ under further hypotheses on $f$. When $f$ is a smooth rotationally symmetric function, sufficient conditions are given in [10] (see also [9] and [23]). For more general smooth functions, there is much interest in the problem (for more details see for example [6], [7], [8], [11] and the references therein). In particular, Chang-Gursky-Yang [8] obtained several results dealing with functions $f$ under sufficient conditions on nondegenerate critical points together with assumptions on the topological degree of a map depending on $f$.

When $f$ is constant, a solution is given by the classical uniformisation theorem. Hamilton [14] provided an elegant way of obtaining the solution in this case, by considering the evolution of the metric $g$ under the Ricci flow:

$$
\partial_{t} g=(r-R) g,
$$

where $r$ is the average value of $R$. He was able to establish the global existence of the flow and its convergence when $r<0$, as well as to solve partially the singular case $M=S^{2}$. This latter case was completely resolved later by Chow [13] (for new approaches see Bartz-Struwe-Ye [4] and also Chen [12] for the Calabi flow). In a recent paper, Struwe [22] gives a unified treatment to the Hamilton-Ricci flow (1.2) and the Calabi flow by using concentrationcompactness methods.

In this paper, we investigate the evolution problem corresponding to the prescribed scalar curvature equation (1.1) when $f$ is not necessarily constant. Without loss of generality, we may suppose that the background metric $g_{0}$ has constant scalar curvature $R_{0}$ (recall that $R_{0}=2 k_{0}$ where $k_{0}$ is the Gaussian curvature; the sign of $k_{0}$ depends only on the topology of $M$ ). A variational approach to problem (1.1) is to consider the functional

$$
J(u)=\int_{M}|\nabla u|^{2} d \mu_{0}+2 k_{0} \int_{M} u d \mu_{0}
$$

on the Sobolev space $H=H^{1}(M)$ under the constraint

$$
u \in X:=\left\{u \in H: L(u)=2 k_{0} \quad \operatorname{Vol}(M)\right\}
$$

where $L: H \rightarrow \mathbb{R}$ is defined by $L(u)=\int_{M} f e^{2 u} d \mu_{0}$. We suppose from now on that $f \in C^{0}(M)$. Since for any $u \in H$ : we have $e^{p u} \in L^{1}\left(M, g_{0}\right)$ for all $p \in \mathbb{R}$ (see [2]), then the function $L$ is well defined on $H$. In order that the set $X$ is non-empty, we will make the following hypothesis on $f$, which is 
necessary in order to solve Problem (1.1). Depending upon the sign of $k_{0}$, we suppose that one of the following conditions is satisfied:

$$
\begin{cases}\text { (i) } \int_{M} f d \mu_{0}<0 & \text { when } k_{0}<0 \\ \text { (ii) } \int_{M} f d \mu_{0}<0 & \text { and } \sup _{x \in M} f(x)>0 \text { when } k_{0}=0 \\ \text { (iii) } \sup _{x \in M} f(x)>0 & \text { when } k_{0}>0 .\end{cases}
$$

Note that when $f \in C^{1}\left(S^{2}\right)$, there is a further necessary condition due to Kazdan-Warner [16] which states that if Problem (1.1) has a solution $u$, then $f$ must satisfy

$$
\int_{S^{2}} \nabla f . \nabla x_{i} e^{2 u} d \mu_{0}=0
$$

for each eigenfunction $x_{i}(1 \leq i \leq 3)$ corresponding to the first eigenvalue of the Laplacian.

The functionals $J$ and $L$ are analytic and their gradients are given respectively by

$$
\langle\nabla J(u), \phi\rangle=2 \int_{M} \nabla u . \nabla \phi d \mu_{0}+2 k_{0} \int_{M} \phi d \mu_{0} \quad \text { for all } \phi \in H,
$$

thus,

$$
\nabla J(u)=2\left(-\Delta_{0}+I\right)^{-1}\left(-\Delta_{0} u+k_{0}\right)
$$

and

$$
\langle\nabla L(u), \phi\rangle=2 \int_{M} f e^{2 u} \phi d \mu_{0} \quad \text { for all } \phi \in H
$$

thus,

$$
\nabla L(u)=2\left(-\Delta_{0}+I\right)^{-1}\left(f e^{2 u}\right)
$$

where $I$ is the identity map of $H$ and $\langle.,$.$\rangle denotes the scalar product on H$.

Since $\nabla L(u) \neq 0$ for all $u \in X$, the set $X$ is a regular hypersurface of $H$. A unit normal is well defined at any point of $u \in X$ and is given by

$$
N(u)=\frac{\nabla L(u)}{\|\nabla L(u)\|},
$$

where $\|$.$\| denotes the norm of H$. This allows us to consider the gradient of the functional $J$ with respect to the hypersurface $X$; this is defined by

$$
\nabla^{X} J(u)=\nabla J(u)-\langle\nabla J(u), N(u)\rangle N(u) .
$$


We now introduce our evolution problem as the negative gradient flow of $J$ with respect to the hypersurface $X$ :

$$
\left\{\begin{array}{l}
\partial_{t} u=-\nabla^{X} J(u) \\
u(0)=u_{0} \in X
\end{array}\right.
$$

If the flow (1.13) exists for all time and converges at infinity, then the limit function $u_{\infty}$ is a solution of (1.1) and so corresponds to a metric of scalar curvature $f$. As such, this flow is a natural geometric deformation and an efficient tool to produce metrics with prescribed scalar curvature in a given conformal class. In this paper we prove the global existence of a solution of (1.13) and show its convergence as $t \rightarrow \infty$ under sufficients conditions on the prescribed scalar curvature $f$. More precisely, our results are as follows:

THEOREM 1. Let $\left(M, g_{0}\right)$ be a compact Riemannian surface without boundary and let $f \in C^{0}(M)$ satisfy the appropriate compatibility condition (1.5). Then for any $u_{0} \in X$, where $X$ is as in (1.4), there exists a unique global solution $u \in C^{\infty}([0, \infty), H)$ of $(1.13)$, satisfying $u(t) \in X$ for all $t \geq 0$. Furthermore, the energy identity

$$
\int_{0}^{t}\left\|\partial_{s} u(s)\right\|^{2} d s+J(u(t))=J\left(u_{0}\right) \quad \text { holds for all } t>0
$$

To prove the convergence of the global solution, we will consider two separate cases: $k_{0} \leq 0$ and $k_{0}>0$. For $k_{0} \leq 0$, our result is as follows:

TheOREM 2. Let $u_{0} \in X$ and $u:[0, \infty) \rightarrow H$ be the solution of $(1.13)$ obtained in Theorem 1.

(i) Suppose that $k_{0}=0$. Then $u$ converges in $H$ to a function $u_{\infty} \in H^{2}(M) \cap$ $C^{1+\alpha}(M)($ for all $0<\alpha<1)$ as $t \rightarrow \infty$. Moreover $u_{\infty}+\lambda$ is a solution of (1.1) for some constant $\lambda$.

(ii) Suppose that $k_{0}<0$. There exists a positive constant $\bar{C}$ depending only on $f^{-}(x)=\sup (-f(x), 0)$ and $M$ such that if $u_{0}$ satisfies

$$
e^{\tau\left\|u_{0}\right\|^{2}} \sup _{x \in M} f(x) \leq \bar{C}
$$

where $\tau>1$ is a constant depending only on $M$ then $u$ converges in $H$ to a solution $u_{\infty} \in H^{2}(M) \cap C^{1+\alpha}(M)$ (for all $0<\alpha<1$ ) of (1.1) as $t \rightarrow \infty$. In particular, if $f \leq 0$, then $u$ converges in $H$ to a solution $u_{\infty} \in H^{2}(M) \cap$ $C^{1+\alpha}(M)($ for all $0<\alpha<1)$ of (1.1) as $t \rightarrow \infty$, for all $u_{0} \in X$.

REMARK 1. We will see in the proof of Part (ii) of Theorem 2 that $\bar{C}=\left(-\inf _{x \in M} f(x)\right) C$ where $C$ is a constant depending on $\operatorname{Vol}(\{x \in M$ : $\left.\left.f(x) \leq \frac{1}{2} \inf _{x \in M} f(x)\right\}\right)$ and $M$. 
Corollary 1.

(i) Suppose that $k_{0}=0$ and let $f \in C^{0}(M)$ satisfy the compatibility condition (1.5)(ii), then Problem (1.1) admits a solution $u \in H^{2}(M) \cap C^{1+\alpha}(M)$ ( for all $0<\alpha<1$ ).

(ii) Suppose that $k_{0}<0$ and let $f \in C^{0}(M)$ be a function which satisfies the compatibility condition (1.5)(i). There exists a positive constant $\bar{C}$ depending only on $f^{-}$and $M$ such that if $f$ satisfies

$$
\sup _{x \in M} f(x) \leq \bar{C},
$$

then Problem (1.1) admits a solution $u \in H^{2}(M) \cap C^{1+\alpha}(M)$ (for all $0<$ $\alpha<1)$. In particular, if $f \leq 0$, then Problem (1.1) admits a solution $u \in$ $H^{2}(M) \cap C^{1+\alpha}(M)($ for all $0<\alpha<1)$.

Part (i) of Corollary 1 can be obtained by a direct variational method as in [1]. When $k_{0}<0$, the variational method fails except for $f<0$. Part (ii) of Corollary 1 has been obtained by Aubin [3] and Bismuth [5] when $f \in C^{\alpha}(M)$ $(0<\alpha<1)$ by using the method of lower and upper solutions together with a fixed point theorem in the Hölder space $C^{2+\alpha}(M)$. In [5], it was shown how the constant $\bar{C}$ depends on $f^{-}$.

We now consider the singular case $k_{0}>0$. Without loss of generality, we may suppose that $M=S^{2}$, with the standard Euclidean metric. We will observe concentration phenomenae; this is because the group of conformal diffeomorphisms of the standard sphere is not compact. By considering functions $f$ invariant under a group $G$ of isometries of $S^{2}$, we will establish convergence.

Recall that a function $f$ on $S^{2}$ is said to be invariant under $G$ or $f$ is $G$-invariant if it satisfies

$$
f(\sigma x)=f(x), \quad \text { for all } \quad x \in S^{2} \quad \text { and } \quad \sigma \in G .
$$

We let $\Sigma$ denote the set of fixed points of $G$, that is

$$
\Sigma=\left\{x \in S^{2}: \sigma x=x \text { for all } \sigma \in G\right\} .
$$

We obtain the following result:

THEOREM 3. Let $f \in C^{0}\left(S^{2}\right)$ be a function invariant under a group $G$ of isometries of $S^{2}$ with $\sup _{x \in S^{2}} f(x)>0$. Let $u_{0} \in X$ be invariant under $G$ and let $u:[0, \infty) \rightarrow H$ be the solution of $(1.13)$ given by Theorem 1 . If either

(i) $\Sigma=\emptyset ;$ or

(ii)

$$
\sup _{P \in \Sigma} f(P) \leq 2 e^{-J\left(u_{0}\right) / 4 \pi}
$$

then $u$ converges in $H$ to a $G$-invariant solution $u_{\infty} \in H^{2}(M) \cap C^{1+\alpha}\left(S^{2}\right)$ (for all $0<\alpha<1$ ) of (1.1) as $t \rightarrow \infty$. 
Let $P \in S^{2}, r>0$, and let $z$ be the coordinate obtained by stereographic projection from $S^{2}-\{P\}$ ( $P$ can be choosen to be the north pole of $S^{2}$ ) to an equatorial plane. We denote by $\phi_{P, r}$ the conformal transformation of $S^{2}$ given by $\phi_{P, r}(z)=r z$. For a suitable choice of the initial data $u_{0}$, we have the following consequence:

COROLlaRY 2. Let $f \in C^{0}\left(S^{2}\right)$ be a function with $\sup _{x \in S^{2}} f(x)>0$ which is invariant under a group $G$ of isometries of $S^{2}$. If either

(i) $\Sigma=\emptyset$; or

(ii) there exist $P_{0} \in \Sigma$ and $r_{0}>0$ satisfying

$$
\sup _{P \in \Sigma} f(P) \leq \frac{1}{4 \pi} \operatorname{Max}\left(0, \int_{S^{2}} f \circ \phi_{P_{0}, r_{0}} d \mu_{0}\right)
$$

then Problem (1.1) admits a $G$-invariant solution $u \in H^{2}\left(S^{2}\right) \cap C^{1+\alpha}\left(S^{2}\right)($ for all $0<\alpha<1)$. In particular if

$$
\sup _{P \in \Sigma} f(P) \leq \frac{1}{4 \pi} \operatorname{Max}\left(0, \int_{S^{2}} f d \mu_{0}\right)
$$

then Problem (1.1) has a G-invariant solution $u \in H^{2}(M) \cap C^{1+\alpha}$ (for all $0<\alpha<1$ ).

Corollary 2 generalises the result of Moser [19] obtained for an even function. It gives a complete study of the problem of prescibed scalar curvature for a function invariant under a group of isometries on $S^{2}$.

REMARK 2.

1) In the above results, $f$ is supposed only continuous. If $f$ is in $C^{2}\left(S^{2}\right)$, then it is easy to see that if $\Delta f(P)>0$ for $P \in \Sigma$ with $f(P)=\sup _{x \in \Sigma} f(x)$, then (1.17) is satisfied.

2) Inequality (1.18) (hence (1.17)) is sharp. For example, let $\epsilon>0$ and take $f(x)=\epsilon x_{3}$. Let $G$ be the group of isometries which fixes the north and south poles of $S^{2}$ then $f$ is $G$-invariant and

$$
\sup _{P \in \Sigma} f(P)=\epsilon, \frac{1}{4 \pi} \int_{S^{2}} f d \mu_{0}=0 .
$$

However, the function $f$ is not the scalar curvature of a metric conformal to $g_{0}$, by the obstruction (1.6) of Kazdan-Warner. 


\section{2. - Global existence}

We set $F(u)=-\nabla^{X} J(u)$. Using (1.11) and (1.12), equation (1.13) becomes

$$
\left\{\begin{array}{l}
\partial_{t} u=F(u)=-\nabla J(u)+\langle\nabla J(u), \nabla L(u)\rangle \frac{\nabla L(u)}{\|\nabla L(u)\|^{2}} \\
u(0)=u_{0} \in X .
\end{array}\right.
$$

Since the functionals $J, L$ are in $C^{\infty}(H)$ and $\nabla L(u) \neq 0$ for all $u \in H$, it follows that $F$ is in $C^{\infty}(H)$. Thus, from the classical Cauchy-Lipschitz theorem, there exists a solution $u \in C^{\infty}([0, T) ; H)$ of equation (2.1) for some $T>0$.

We now show that the solution $u$ is globally defined on $[0, \infty)$. Rewrite $\nabla J(u)$, given by (1.8), in the form

$$
\nabla J(u)=-2\left(\left(-\Delta_{0}+I\right)^{-1}-I\right) u+2 k_{0} .
$$

From (2.1), we observe that

$$
\|F(u)\| \leq 2\|\nabla J(u)\| .
$$

Using the fact that $\left(-\Delta_{0}+I\right)^{-1}: H \rightarrow H$ is a continuous linear map, we deduce from (2.2) and (2.3) that

$$
\|F(u)\| \leq C\|u\|+C .
$$

It follows from inequality (2.4) that

$$
\partial_{t}\|u\|^{2} \leq C_{1}\|u\|^{2}+C_{1},
$$

where $C_{1}$ is a positive constant. By integrating (2.5) between 0 and $t(t<T)$, we obtain

$$
\|u(t)\| \leq\left\|u_{0}\right\| e^{C_{1} T / 2}+e^{C_{1} T / 2} .
$$

Inequality (2.6) guarantees that the solution $u$ may be extended for all time.

Next, on taking the inner product of (2.1) with $\nabla L(u)$, we see that

$$
\partial_{t} L(u)=\left\langle\nabla L(u), \partial_{t} u\right\rangle=0 .
$$

Thus, for all $t \geq 0$ we have $L(u(t))=L\left(u_{0}\right)=2 k_{0} \operatorname{Vol}(M)$, so that $u(t) \in X$ for all $t \geq 0$. To complete the proof of Theorem 1 , it remains to prove that $u$ satisfies the energy inequality (1.14). On taking the inner product of (1.13) with $\partial_{t} u$, we obtain

$$
\left\|\partial_{t} u\right\|^{2}=-\left\langle\nabla J(u), \partial_{t} u\right\rangle+\langle\nabla J(u), N(u)\rangle\left\langle N(u), \partial_{t} u\right\rangle .
$$

Since

we deduce from (2.7) that

$$
\left\langle N(u), \partial_{t} u\right\rangle=\frac{\partial_{t} L(u)}{\|\nabla L(u)\|}=0,
$$

$$
\left\|\partial_{t} u\right\|^{2}=-\left\langle\nabla J(u), \partial_{t} u\right\rangle=-\partial_{t} J(u) .
$$

Integrating (2.8) between 0 and $t$, we obtain inequality (1.14). This completes the proof of Theorem 1 . 


\section{3. - Uniform bounds}

We prove that if the solution $u$ of equation (1.13) is uniformly bounded in $H$ with respect to time, then $u$ converges at infinity. More precisily,

Proposition. Let $u:[0, \infty) \rightarrow H$ be the solution of (1.13) obtained in Theorem 1. Suppose that for all $t>0, u$ satisfies

$$
\|u(t)\| \leq C,
$$

where $C$ is a positive constant. Then $u(t)$ converges in $H$ to a function $u_{\infty} \in$ $H^{2}(M) \cap C^{1+\alpha}(M)(0<\alpha<1)$ as $t \rightarrow \infty$. Moreover, if $k_{0} \neq 0$ then $u_{\infty}$ is a solution of (1.1). And if $k_{0}=0$, then $u_{\infty}+\lambda$ is a solution of (1.1) for some constant $\lambda$.

Proof. The energy inequality (1.14) and (3.1) imply

$$
\int_{0}^{\infty}\left\|\partial_{s} u(s)\right\|^{2} d s \leq J\left(u_{0}\right)+C_{1},
$$

where $C_{1}$ is a positive constant. Thus, there exists a sequence $t_{k} \rightarrow \infty$ such that

$$
\left\|\partial_{t} u\left(t_{k}\right)\right\|=\left\|\nabla^{X} J\left(u\left(t_{k}\right)\right)\right\| \rightarrow 0 .
$$

From (3.1), we have $\left\|u\left(t_{k}\right)\right\| \leq C$, so there exist a function $u_{\infty} \in H$ and a subsequence of $t_{k}$ (that we also call $t_{k}$ ), such that

$$
\begin{cases}u\left(t_{k}\right) \rightarrow u_{\infty} & \text { weakly in } H \\ u\left(t_{k}\right) \rightarrow u_{\infty} & \text { strongly in } L^{2}(M),\end{cases}
$$

and by the Moser-Trudinger inequality [18]

$$
\int_{M} e^{p u\left(t_{k}\right)} d \mu_{0} \leq C_{p} \quad \text { for all } \quad p \in \mathbb{R},
$$

where $C_{p}$ is a positive constant. We first show that $u_{\infty} \in X$. Using (3.3) and (3.4), a computation shows that

$$
\lim _{k \rightarrow \infty} \int_{M} f e^{2 u\left(t_{k}\right)} d \mu_{0}=\int_{M} f e^{2 u_{\infty}} d \mu_{0} .
$$

Since $u\left(t_{k}\right) \in X$ : that is $\int_{M} f e^{2 u\left(t_{k}\right)} d \mu_{0}=2 k_{0} \operatorname{Vol}(M)$, we deduce from (3.5) that $u_{\infty} \in X$.

Now, we prove that $\nabla^{X} J\left(u_{\infty}\right)=0$. Recall that

$$
\nabla^{X} J(u(t))=\nabla J(u(t))-\langle\nabla J(u(t)), \nabla L(u(t))\rangle \frac{\nabla L(u(t))}{\|\nabla L(u(t))\|^{2}},
$$


with

$$
\nabla J(u(t))=2\left(-\Delta_{0}+I\right)^{-1}\left(-\Delta_{0} u(t)+k_{0}\right)=-2\left(\left(-\Delta_{0}+I\right)^{-1}-I\right) u(t)+2 k_{0},
$$

and

$$
\nabla L(u(t))=2\left(-\Delta_{0}+I\right)^{-1}\left(f e^{2 u(t)}\right) .
$$

Using (3.3), (3.5) and since $\left(-\Delta_{0}+I\right)^{-1}: H \rightarrow H$ is a compact operator, it is not difficult to show from (3.6), (3.7), (3.8) that $\nabla^{X} J\left(u\left(t_{k}\right)\right)$ converges weakly in $H$ to $\nabla^{X} J\left(u_{\infty}\right)$ and

$$
\lim _{k \rightarrow \infty}\left\|u\left(t_{k}\right)-u_{\infty}\right\|=0
$$

It follows from (3.2) that $\nabla^{X} J\left(u_{\infty}\right)=0$. Hence, we have

$$
\left(-\Delta_{0}+I\right)^{-1}\left(-\Delta_{0} u_{\infty}+k_{0}\right)=\eta\left(u_{\infty}\right)\left(-\Delta_{0}+I\right)^{-1}\left(f e^{2 u_{\infty}}\right)
$$

where $\eta\left(u_{\infty}\right)$ is a constant. Hence,

$$
-\Delta_{0} u_{\infty}+k_{0}=\eta\left(u_{\infty}\right) f e^{2 u_{\infty}}
$$

A standard elliptic argument gives $u_{\infty} \in H^{2}(M) \cap C^{1+\alpha}(M)$ (for all $0<\alpha<$ 1). If $k_{0} \neq 0$ : since $u_{\infty} \in X$, by integrating (3.10) on $M$, we deduce that $\eta\left(u_{\infty}\right)=\frac{1}{2}$. Thus $u_{\infty}$ is a solution of (1.1).

If $k_{0}=0$, then $\eta\left(u_{\infty}\right) \neq 0$, otherwise from (3.10), $u_{\infty}$ is a constant map; since $u_{\infty} \in X$, it follows that $\int_{M} f d \mu_{0}=0$ and this contradicts the hypothesis (1.5)(ii). Now, if we multiply (3.10) by $e^{-2 u_{\infty}}$ and we integrate on $M$, then from (1.5)(ii) we observe that $\eta\left(u_{\infty}\right)>0$. And it is easy to see that $v_{\infty}=u_{\infty}+\frac{1}{2} \log \left(2 \eta\left(u_{\infty}\right)\right)=u_{\infty}+\lambda\left(u_{\infty}\right)$ is a solution of $(1.1)$.

It remains to prove that $\lim _{t \rightarrow \infty}\left\|u(t)-u_{\infty}\right\|=0$. To this end, we need the following version of the so-called Lojasiewicz-Simon inequality.

Lemma 1. Let $X$ be an analytic manifold modelled on a Hilbert space $\mathcal{H}$ and suppose that $G: X \rightarrow \mathbb{R}$ is an analytic function on a neighborhood of a point $\bar{u} \in X$ satisfying

(i) $\nabla G(\bar{u})=0$,

(ii) $\nabla^{2} G(\bar{u}): T_{\bar{u}} X \rightarrow T_{\bar{u}} X$ has finite dimensional kernel.

Here, $\nabla G$ denotes the gradient in $X$ of $G$ and we have identified the second derivative $d^{2} G(\bar{u}): T_{\bar{u}} X \times T_{\bar{u}} X \rightarrow \mathbb{R}$ with the linear map $\nabla^{2} G(\bar{u}): T_{\bar{u}} X \rightarrow T_{\bar{u}} X$. Then, there are constants $\sigma>0$ and $0<\theta<\frac{1}{2}$ such that if $u \in B(\bar{u}, \sigma)$, where $B(\bar{u}, \sigma)$ is the geodesic ball of radius $\sigma$ centered at $\bar{u}$, then

$$
\|\nabla G(\bar{u})\| \geq|G(u)-G(\bar{u})|^{1-\theta} .
$$


Proof of Lemma 1. The proof follows closely the proof of Theorem 3 of [21]. It suffices to take an analytic chart $\phi: U \rightarrow V$ where $U$ is a neighborhood of $\bar{u}$ and $V$ is a neighborhood of 0 in $\mathcal{H}$ with $\phi(\bar{u})=0$. The functional $\bar{G}=G \circ \phi^{-1}: V \subset \mathcal{H} \rightarrow \mathbb{R}$ is analytic in a neighborhood of 0 and condition (i) gives $\nabla \bar{G}(0)=0$ (here $\nabla \bar{G}$ is the gradient of $\bar{G}$ in $\mathcal{H}$ ). Using condition (ii), we adapt the proof of [21] to $\bar{G}$. So there are constants $\sigma>0$ and $0<\theta<\frac{1}{2}$ such that if $\|x\|<\sigma$ the functional $\bar{G}$ satisfy

$$
\|\nabla \bar{G}(0)\| \geq|\bar{G}(x)-\bar{G}(0)|^{1-\theta},
$$

and the proof of Lemma 1 follows immediately. We omit the details.

By using Lemma 1, we will show that the functional $J$ satisfies the inequality (3.11) with $\bar{u}=u_{\infty}$. Since $L$ is an analytic function on $H$ then $X$ is an analytic manifold. The functional $J: X \subset H \rightarrow \mathbb{R}$ is analytic and $\nabla^{X} J\left(u_{\infty}\right)=0$. Let $\Pi_{u_{\infty}}: H \rightarrow T_{u_{\infty}} X$ be the projection onto $T_{u_{\infty}} X$. Using formulae (3.6), (3.7) and (3.8), a straightforward computation gives for all $v \in T_{u_{\infty}} X$ :

$$
\nabla^{2} J\left(u_{\infty}\right)(v)=2 v+\Pi_{u_{\infty}}(T(v)),
$$

with

$$
\begin{aligned}
T(v)= & -2\left(-\Delta_{0}+I\right)^{-1}(v)-4\left\langle\nabla J\left(u_{\infty}\right), \frac{\nabla L\left(u_{\infty}\right)}{\| \nabla L\left(u_{\infty} \|^{2}\right.}\right\rangle\left(-\Delta_{0}+I\right)^{-1}\left(f e^{2 u_{\infty}} v\right) \\
& +\left\langle\nabla J\left(u_{\infty}\right), \frac{\nabla L\left(u_{\infty}\right)}{\| \nabla L\left(u_{\infty} \|^{2}\right.}\right\rangle\left\langle v, \frac{\nabla\left(\left\|\nabla L\left(u_{\infty}\right)\right\|\right)}{\left\|\nabla L\left(u_{\infty}\right)\right\|}\right\rangle \nabla L\left(u_{\infty}\right) .
\end{aligned}
$$

It easy to check that $T$ is a compact operator. Since $\Pi_{u_{\infty}}$ is a continuous map, it follows that $\Pi_{u_{\infty}} \circ T$ is also compact. Thus, we deduce that the kernel of $\nabla^{2} J\left(u_{\infty}\right)$ has finite dimension. By Lemma 1, there are constants $\sigma>0$ and $0<\theta<\frac{1}{2}$ such that if $\left\|u-u_{\infty}\right\|<\sigma$ then

$$
\left\|\nabla^{X} J(u)\right\| \geq\left|J(u)-J\left(u_{\infty}\right)\right|^{1-\theta} .
$$

We are now in a position to prove that $\lim _{t \rightarrow \infty}\left\|u(t)-u_{\infty}\right\|=0$ as in [15] and [21]. From (3.9), we see that for all $\epsilon>0$ there exists $N>0$ such that

$$
\left\|u\left(t_{n}\right)-u_{\infty}\right\|<\frac{\epsilon}{2} \quad \text { and } \quad \frac{1}{\theta}\left(J\left(u\left(t_{n}\right)-J\left(u_{\infty}\right)\right)^{\theta}<\frac{\epsilon}{2} \quad \text { for all } n \geq N .\right.
$$

Let $t^{*}=\sup \left\{t \geq t_{N}:\left\|u(s)-u_{\infty}\right\|<\sigma\right.$ for all $\left.s \in\left[t_{N}, t\right]\right\}$. Suppose that $t^{*}<\infty$. If there exists $\bar{t}$ such that $J(u(\bar{t}))=J\left(u_{\infty}\right)$ then since $J$ is noincreasing and from the uniqueness of the solution $u$ of (1.13), it follows that $u(t) \equiv u_{\infty}$ for all $t \geq \bar{t}$. So the solution is stationary. Otherwise, we obtain for all $t \in\left[t_{N}, t^{*}\right]$

$$
-\partial_{t}\left\{J(u(t))-J\left(u_{\infty}\right)\right\}^{\theta}=-\theta \partial_{t} J(u(t))\left\{J(u(t))-J\left(u_{\infty}\right)\right\}^{\theta-1} .
$$


From (2.8), we have

$$
-\partial_{t} J(u(t))=\left\|\partial_{t} u(t)\right\|\left\|\nabla^{X} J(u(t))\right\| .
$$

If we put (3.14) into (3.13) and use the estimate (3.12), we obtain

$$
-\partial_{t}\left\{J\left(u(t)-J\left(u_{\infty}\right)\right\}^{\theta} \geq \theta\left\|\partial_{t} u(t)\right\| .\right.
$$

Integrating inequality (3.15) between $t_{N}$ and $t^{*}$, since $J$ is noincreasing, we deduce

$$
\int_{t_{N}}^{t^{*}}\left\|\partial_{t} u(t)\right\| d t \leq \frac{1}{\theta}\left(J\left(u\left(t_{N}\right)\right)-J\left(u_{\infty}\right)\right)^{\theta}<\frac{\epsilon}{2} .
$$

But from (3.16) and for sufficiently small $\epsilon$, we have

$$
\left\|u\left(t^{*}\right)-u_{\infty}\right\| \leq \int_{t_{N}}^{t^{*}}\left\|\partial_{t} u(t)\right\| d t+\left\|u\left(t_{N}\right)-u_{\infty}\right\| \leq \epsilon<\sigma .
$$

This contradicts the definition of $t^{*}$, hence $t^{*}=\infty$. So, from (3.16) we obtain

$$
\int_{t_{N}}^{\infty}\left\|\partial_{t} u(t)\right\| d t \leq \frac{\epsilon}{2} .
$$

It follows immediately that $\left\|u(t)-u_{\infty}\right\| \leq \epsilon$ for all $t \geq t_{N}$. This completes the proof of the proposition.

\section{4. - Convergence}

To obtain convergence, we have to prove uniform boundedness in $H$ of the global solution $u:[0, \infty) \rightarrow H$ of (1.13) obtained in Theorem 1 .

The Null CASE. From the energy inequality (1.14), we deduce that $J(u(t)) \leq$ $J\left(u_{0}\right)$ for all $t \geq 0$. Since $k_{0}=0$, it follows that

$$
\int_{M}|\nabla u(t)|^{2} d \mu_{0} \leq \int_{M}\left|\nabla u_{0}\right|^{2} d \mu_{0} .
$$

To show that $u$ is uniformly bounded in $H$, it remains to prove, that for all $t \geq 0$, $\int_{M} u^{2}(t) d \mu_{0} \leq C$ where $C$ is a positive constant. On taking the inner product of (1.13) with the constant function 1 and using formulae (1.11) and (1.12), we obtain

$$
\begin{aligned}
\left\langle\partial_{t} u(t), 1\right\rangle= & -\langle\nabla J(u(t)), 1\rangle \\
& +\frac{1}{\|\nabla L(u(t))\|^{2}}\langle\nabla J(u(t)), \nabla L(u(t))\rangle\langle\nabla L(u(t)), 1\rangle .
\end{aligned}
$$


Since $k_{0}=0$ and $u(t) \in X$ for all $t \geq 0$, it is easy to see from (1.7) and (1.9), that $\langle\nabla J(u(t)), 1\rangle=0$ and $\langle\nabla L(u(t)), 1\rangle=0$. So (4.2) implies

$$
\left\langle\partial_{t} u(t), 1\right\rangle=\int_{M} \nabla \partial_{t} u(t) \nabla 1 d \mu_{0}+\int_{M} \partial_{t} u(t) 1 d \mu_{0}=0,
$$

thus

$$
\int_{M} u(t, .) d \mu_{0} \equiv \text { Cste for all } t \geq 0 .
$$

We recall Poincaré's inequality

$$
\int_{M} u^{2} d \mu_{0} \leq \frac{1}{\lambda_{1}} \int_{M}|\nabla u|^{2} d \mu_{0}+\frac{1}{\operatorname{Vol}(M)}\left(\int_{M} u d \mu_{0}\right)^{2},
$$

where $\lambda_{1}$ is the first eigenvalue of $\Delta_{0}$. Combining (4.1), (4.3), and (4.4), we deduce that, for all $t \geq 0$

$$
\int_{M} u^{2}(t) d \mu_{0} \leq C
$$

where $C$ is a positive constant depending on $M$ and $u_{0}$. This completes the proof of Part (i) of Theorem 2.

The NegAtive CASE. Without loss of generality, we may suppose that $k_{0}=-1$. The proof of Part (ii) of Theorem 2 is essentially based on the following non-concentration lemma.

LeMma 2. Let $K$ be any measurable subset of $M$ with $\operatorname{Vol}(K)>0$. Then there exists a constant $C_{K} \geq 1$ depending on $M$ and $\operatorname{Vol}(K)$ such that the global solution $u:[0, \infty) \rightarrow H$ of $(\overline{1.13})$ satisfies for all $t \geq 0$

$$
\int_{M} e^{2 u(t)} d \mu_{0} \leq C_{K} e^{\alpha\left\|u_{0}\right\|^{2}} \operatorname{Max}\left(\left(\int_{K} e^{2 u(t)} d \mu_{0}\right)^{\alpha}, 1\right),
$$

where $\alpha>1$ is a constant depending only on $M$.

Proof of Lemma 2. We fix $t \geq 0$. To prove inequality (4.5), we first establish the following estimate

$$
\int_{M} u d \mu_{0} \leq \mid J\left(u_{0} \mid+\frac{C}{\operatorname{Vol}(K)}+\frac{4 \operatorname{Vol}(M)}{\operatorname{Vol}(K)} \operatorname{Max}\left(\int_{K} u d \mu_{0}, 0\right),\right.
$$

where $C>0$ is a constant depending on $M$.

We may suppose that $\int_{M} u d \mu_{0}>0$, otherwise the estimate (4.6) is trivial. The energy inequality (1.14) implies

$$
\int_{M}|\nabla u|^{2} d \mu_{0} \leq J\left(u_{0}\right)+2 \int_{M} u d \mu_{0} .
$$


Combining (4.7) and the Poincaré inequality (4.4), we have

$$
\int_{M} u^{2} d \mu_{0} \leq \frac{1}{\lambda_{1}} J\left(u_{0}\right)+\frac{2}{\lambda_{1}} \int_{M} u d \mu_{0}+\frac{1}{\operatorname{Vol}(M)}\left(\int_{M} u d \mu_{0}\right)^{2} .
$$

We first suppose that $\int_{K} u d \mu_{0} \leq 0$. Since $\int_{M} u d \mu_{0}>0$, we have

$$
\left(\int_{M} u d \mu_{0}\right)^{2} \leq\left(\int_{K^{c}} u d \mu_{0}\right)^{2} \leq \operatorname{Vol}\left(K^{c}\right) \int_{M} u^{2} d \mu_{0}
$$

where $K^{c}=M \backslash K$. Thus, by putting this last inequality into (4.8), we obtain

$$
\frac{\operatorname{Vol}(K)}{\operatorname{Vol}(M)} \int_{M} u^{2} d \mu_{0} \leq \frac{1}{\lambda_{1}} J\left(u_{0}\right)+\frac{2}{\lambda_{1}} \int_{M} u d \mu_{0} .
$$

By Young's inequality

$$
\frac{2}{\lambda_{1}} \int_{M} u d \mu_{0} \leq \frac{\operatorname{Vol}(K)}{2 \operatorname{Vol}(M)} \int_{M} u^{2} d \mu_{0}+\frac{2 \operatorname{Vol}^{2}(M)}{\lambda_{1}^{2} \operatorname{Vol}(K)} .
$$

If we put (4.10) into (4.9), we deduce that

$$
\int_{M} u^{2} d \mu_{0} \leq \frac{2 \operatorname{Vol}(M)}{\lambda_{1} \operatorname{Vol}(K)}\left|J\left(u_{0}\right)\right|+\frac{4 \operatorname{Vol}^{3}(M)}{\lambda_{1}^{2} \operatorname{Vol}^{2}(K)} .
$$

From (4.11), it follows that

$$
\left(\int_{M} u d \mu_{0}\right)^{2} \leq \frac{2 \operatorname{Vol}^{2}(M)}{\lambda_{1} \operatorname{Vol}(K)}\left|J\left(u_{0}\right)\right|+\frac{4 \operatorname{Vol}^{4}(M)}{\lambda_{1}^{2} \operatorname{Vol}^{2}(K)} .
$$

Since

$$
\frac{2 \operatorname{Vol}^{2}(M)}{\lambda_{1} \operatorname{Vol}(K)}\left|J\left(u_{0}\right)\right| \leq\left|J\left(u_{0}\right)\right|^{2}+\frac{\operatorname{Vol}^{4}(M)}{\lambda_{1}^{2} \operatorname{Vol}^{2}(K)},
$$

by combining (4.12) and (4.13), we obtain

$$
\int_{M} u d \mu_{0} \leq \mid J\left(u_{0} \mid+\frac{C}{\operatorname{Vol}(K)},\right.
$$

where $C>0$ is a constant depending on $M$; hence the estimate (4.6) is established.

We now suppose that $\int_{K} u d \mu_{0}>0$. From (4.8), we obtain

$$
\begin{aligned}
& \frac{\operatorname{Vol}(K)}{\operatorname{Vol}(M)} \int_{M} u^{2} d \mu_{0} \leq \frac{1}{\lambda_{1}} J\left(u_{0}\right)+\frac{2}{\lambda_{1}} \int_{M} u d \mu_{0} \\
& +\frac{1}{\operatorname{Vol}(M)}\left(\int_{K} u d \mu_{0}\right)^{2}+\frac{2}{\operatorname{Vol}(M)}\left(\int_{K} u d \mu_{0}\right)\left(\int_{K^{c}} u d \mu_{0}\right) .
\end{aligned}
$$


Using Young's inequality, we deduce

$$
\begin{aligned}
& \frac{2}{\operatorname{Vol}(M)}\left(\int_{K} u d \mu_{0}\right)\left(\int_{K^{c}} u d \mu_{0}\right) \\
& \leq \frac{2 \operatorname{Vol}\left(K^{c}\right)}{\operatorname{Vol}(K) \operatorname{Vol}(M)}\left(\int_{K} u d \mu_{0}\right)^{2}+\frac{\operatorname{Vol}(K)}{2 \operatorname{Vol}(M)}\left(\int_{K^{c}} u^{2} d \mu_{0}\right) .
\end{aligned}
$$

Combining (4.15) and (4.16), we obtain

(4.17) $\frac{\operatorname{Vol}(K)}{2 \operatorname{Vol}(M)} \int_{M} u^{2} d \mu_{0} \leq \frac{1}{\lambda_{1}} J\left(u_{0}\right)+\frac{2}{\lambda_{1}} \int_{M} u d \mu_{0}+\frac{3}{\operatorname{Vol}(K)}\left(\int_{K} u d \mu_{0}\right)^{2}$.

By Young's inequality once more

$$
\frac{2}{\lambda_{1}} \int_{M} u d \mu_{0} \leq \frac{\operatorname{Vol}(K)}{4 \operatorname{Vol}(M)} \int_{M} u^{2} d \mu_{0}+\frac{4 \operatorname{Vol}^{2}(M)}{\lambda_{1}^{2} \operatorname{Vol}(K)},
$$

thus, by (4.17)

$$
\int_{M} u^{2} d \mu_{0} \leq \frac{4 \operatorname{Vol}(M)}{\lambda_{1} \operatorname{Vol}(K)} \mid J\left(u_{0} \mid+\frac{16 \operatorname{Vol}^{3}(M)}{\lambda_{1}^{2} \operatorname{Vol}^{2}(K)}+\frac{12 \operatorname{Vol}(M)}{\operatorname{Vol}^{2}(K)}\left(\int_{K} u d \mu_{0}\right)^{2}\right.
$$

It is clear that (4.18) gives

$$
\int_{M} u d \mu_{0} \leq\left|J\left(u_{0}\right)\right|+\frac{C}{\operatorname{Vol}(K)}+\frac{4 \operatorname{Vol}(M)}{\operatorname{Vol}(K)} \int_{K} u d \mu_{0}
$$

where $C>0$ is a constant depending on $M$. Estimate (4.6) is therefore established.

Let us prove inequality (4.5). We recall Aubin's inequality (see [2]):

$$
\int_{M} e^{2 u} d \mu_{0} \leq C \exp \left(\beta \int_{M}|\nabla u|^{2} d \mu_{0}+\frac{2}{\operatorname{Vol}(M)} \int_{M} u d \mu_{0}\right),
$$

where $C$ and $\beta$ are two positive constants depending on $M$. In view of (4.7), inequality (4.19) becomes

$$
\begin{aligned}
\int_{M} e^{2 u} d \mu_{0} & \leq C \exp \left(\beta J\left(u_{0}\right)+2\left(\beta+\frac{1}{\operatorname{Vol}(M)}\right) \int_{M} u d \mu_{0}\right) \\
& \leq C \exp \left(A\left\|u_{0}\right\|^{2}+B \int_{M} u d \mu_{0}\right)
\end{aligned}
$$

where $A, B$ and $C$ are positive constants depending only on $M$.

From (4.6) and (4.20), we deduce that

$$
\int_{M} e^{2 u} d \mu_{0} \leq C_{K} \exp \left(A_{1}\left\|u_{0}\right\|^{2}+\frac{B_{1}}{\operatorname{Vol}(K)} \operatorname{Max}\left(\int_{K} u d \mu_{0}, 0\right)\right),
$$

where $C_{K} \geq 1$ is a constant depending on $M$ and $\operatorname{Vol}(K)$, and $A_{1}, B_{1}$ are positive constants depending only on $M$.

Using Jensen's inequality, (4.21) gives the estimate (4.5) of Lemma 2. 
Now, estimate (4.5) in Lemma 2 allows us to bound uniformly $\int_{M} e^{2 u(t)} d \mu_{0}$. Let $f^{+}(x)=\sup (f(x), 0)$ and $K=\left\{x \in M: f(x) \leq \frac{1}{2} \inf _{x \in M} f(x)\right\}$.

Since for all $t \geq 0, u(t) \in X$, we have

$$
2 \operatorname{Vol}(M)=\int_{M}-f e^{2 u(t)} d \mu_{0}=\int_{M} f^{-} e^{2 u(t)} d \mu_{0}-\int_{M} f^{+} e^{2 u(t)} d \mu_{0},
$$

which gives, setting $t=0$

$$
\frac{2 \operatorname{Vol}(M)}{\left(-\inf _{x \in M} f(x)\right)} \leq \int_{M} e^{2 u_{0}} d \mu_{0}
$$

From Aubin's inequality (4.19), we have the estimate

$$
\int_{M} e^{2 u_{0}} d \mu_{0} \leq C_{1} e^{C_{1}\left\|u_{0}\right\|^{2}}
$$

where $C_{1}>1$ depends on $M$. Hence

$$
\frac{2 \operatorname{Vol}(M)}{\left(-\inf _{x \in M} f(x)\right)} \leq C_{1} e^{C_{1}\left\|u_{0}\right\|^{2}}
$$

Now, let

$$
\gamma=C_{K}\left(8 C_{1}\right)^{\alpha} e^{\left(C_{1}+1\right) \alpha\left\|u_{0}\right\|^{2}} \quad \text { and } \quad \tau=\alpha\left(C_{1}+1\right)-C_{1},
$$

where $C_{K} \geq 1$ and $\alpha>1$ are the constants in Lemma 2. Let us make precise the hypothesis (1.15) of Theorem 2. We suppose that $u_{0}$ satisfies:

$$
e^{\tau\left\|u_{0}\right\|^{2}} \sup _{x \in M} f(x) \leq \bar{C}
$$

where we take $\bar{C}=-\inf _{x \in M} f(x) /\left(8^{\alpha} C_{K} C_{1}^{\alpha-1}\right)$. Under (4.25) we shall prove that for all $t \geq 0$,

$$
\int_{M} e^{2 u(t)} d \mu_{0} \leq 2 \gamma
$$

Set $I=\left\{t \geq 0: \int_{M} e^{2 u(s)} d \mu_{0} \leq 2 \gamma\right.$ for all $\left.s \in[0, t]\right\}$. From (4.23) it follows that $I \neq \varnothing$ since $0 \in I$. Let $T=\sup I$. Suppose that $T<\infty$; from the continuity of the map $t \rightarrow \int_{M} e^{2 u(t)} d \mu_{0}$, it follows that

$$
\int_{M} e^{2 u(T)} d \mu_{0}=2 \gamma
$$


We distinguish two cases: either $\int_{M} f^{+} e^{2 u(T)} d \mu_{0} \leq \frac{1}{2} \int_{M} f^{-} e^{2 u(T)} d \mu_{0}$ or $\int_{M} f^{+} e^{2 u(T)} d \mu_{0}>\frac{1}{2} \int_{M} f^{-} e^{2 u(T)} d \mu_{0}$. In the first case, from (4.22) we have

$$
\int_{M} f^{-} e^{2 u(T)} d \mu_{0} \leq 8 \operatorname{Vol}(M) .
$$

Since $f^{-}(x) \geq \frac{1}{2}\left(-\inf _{x \in M} f(x)\right)$ for all $x \in K$, (4.28) implies

$$
\int_{K} e^{2 u(T)} d \mu_{0} \leq \frac{16 \operatorname{Vol}(M)}{\left(-\inf _{x \in M} f(x)\right)} .
$$

Combining (4.24) and (4.29), we obtain

$$
\int_{K} e^{2 u(T)} d \mu_{0} \leq 8 C_{1} e^{C_{1}\left\|u_{0}\right\|^{2}}
$$

Applying Lemma 2, from (4.30), we get

$$
\int_{M} e^{2 u(T)} d \mu_{0} \leq \gamma
$$

which contradicts (4.27). Now suppose that we are in the case $\int_{M} f^{+} e^{2 u(T)} d \mu_{0}>$ $\frac{1}{2} \int_{M} f^{-} e^{2 u(T)} d \mu_{0}$. Then

$\frac{\left(-\inf _{x \in M} f(x)\right)}{2} \int_{K} e^{2 u(T)} d \mu_{0} \leq \int_{M} f^{-} e^{2 u(T)} d \mu_{0} \leq 2 \int_{M} f^{+} e^{2 u(T)} d \mu_{0} \leq 4 \gamma \sup _{x \in M} f(x)$,

thus

$$
\int_{K} e^{2 u(T)} d \mu_{0} \leq \frac{8 \gamma \sup _{x \in M} f(x)}{\left(-\inf _{x \in M} f(x)\right)} .
$$

Combining (4.31) and (4.25), we get

$$
\int_{K} e^{2 u(T)} d \mu_{0} \leq 8 C_{1} e^{C_{1}\left\|u_{0}\right\|^{2}},
$$

which gives, by applying Lemma 2,

$$
\int_{M} e^{2 u(T)} d \mu_{0} \leq \gamma
$$

contradicting (4.27) once more. Thus $T=\infty$, and we have established (4.26).

Estimate (4.26) allows us to bound uniformly $u$ in $H$. Using Jensen's inequality, (4.26) implies that

$$
\int_{M} u(t) d \mu_{0} \leq C
$$


where $C>0$ depends on $M, \operatorname{Vol}(K)$ and $u_{0}$. Combining (4.7) and (4.32), we find that

$$
\int_{M}|\nabla u(t)|^{2} d \mu_{0} \leq C
$$

with $C>0$ depending on $M, \operatorname{Vol}(K)$ and $u_{0}$. From the energy inequality (4.7) and (4.32), we have

$$
\left|\int_{M} u(t) d \mu_{0}\right| \leq C,
$$

with $C>0$ depending on $M, \operatorname{Vol}(K)$ and $u_{0}$. We deduce from (4.33), (4.34) and the Poincaré inequality (4.4) that

$$
\int_{M} u^{2}(t) d \mu_{0} \leq C,
$$

with $C>0$ depending on $M, \operatorname{Vol}(K)$ and $u_{0}$. Thus, by (4.33) and (4.35), we conclude that for all $t \geq 0:\|u(t)\| \leq C$ ste. This completes the proof of Part (ii) of Theorem 2.

The POSITIVe CASE. For any $P \in S^{2}$ and $r \geq 1$, we set $v_{P, r}=u \circ \phi_{P, r}+$ $\frac{1}{2} \log \left(\operatorname{det} d \phi_{P, r}\right)$. Recall that $\phi_{P, r}$ is the conformal transformation on $S^{2}$ given by $\phi_{P, r}(z)=r z$, where $z$ is the coordinate obtained by stereographic projection from $P$. We first notice that

$$
J\left(v_{P, r}\right)=J(u) \leq J\left(u_{0}\right)
$$

and

$$
\int_{S^{2}} f \circ \phi_{P, r} e^{2 v_{P, r}} d \mu_{0}=\int_{S^{2}} f e^{2 u} d \mu_{0}=8 \pi .
$$

From (4.37), it follows that

$$
\int_{S^{2}} e^{2 v_{P}, r} d \mu_{0} \geq C,
$$

where $C$ is a positive constant depending on $\sup _{x \in S^{2}} f(x)$. For all $t \geq 0$, there exist $P(t) \in S^{2}$ and $r(t) \geq 1$ (see [6]) such that

$$
\int_{S^{2}} x_{i} e^{2 v_{P(t), r(t)}} d \mu_{0}=0 \quad \text { for } \quad i=1,2,3 .
$$

From now on, we set $v(t)=v_{P(t), r(t)}$ and $\phi(t)=\phi_{P(t), r(t)}$. By a result of Aubin [2], in view of (4.38) and (4.39), for any $\epsilon>0$, there exists a constant $C_{\epsilon}$ such that

$$
C \leq \frac{1}{4 \pi} \int_{S^{2}} e^{2 v(t)} d \mu_{0} \leq C_{\epsilon} \exp \left(\frac{(1 / 2+\epsilon)}{4 \pi} \int_{S^{2}}|\nabla v(t)|^{2} d \mu_{0}+\frac{2}{4 \pi} \int_{S^{2}} v(t) d \mu_{0}\right)
$$


Combining (4.40) and (4.36), we deduce

$$
\int_{S^{2}}|\nabla v(t)|^{2} d \mu_{0} \leq C
$$

and

$$
\left|\int_{S^{2}} v(t) d \mu_{0}\right| \leq C,
$$

where $C$ is a positive constant depending on $u_{0}$ and $\sup _{x \in S^{2}} f(x)$. Thus, by the Moser-Trudinger inequality [18], estimates (4.41) and (4.42) show that

$$
\int_{S^{2}} e^{|4 v(t)|} d \mu_{0} \leq \text { Cste. }
$$

From (4.43), it follows that

$$
\int_{S^{2}} v^{2}(t) d \mu_{0} \leq \text { Cste }
$$

which implies with (4.41) that

$$
\|v(t)\| \leq \text { Cste }
$$

Now, in order to prove that $u$ is uniformly bounded in $H$, we need the following concentration lemma.

LEMMA 3. Either

(i) there exists a constant $C$ such that $\|u(t)\| \leq C$ or

(ii) there exist a sequence $t_{n} \rightarrow \infty$ and $P_{\infty} \in \bar{S}^{2}$ such that for all $r>0$

$$
\lim _{n \rightarrow \infty} \int_{B\left(P_{\infty}, r\right)} f e^{2 u\left(t_{n}\right)} d \mu_{0}=8 \pi .
$$

Proof of Lemma 3. We follow the ideas of Chang-Yang [6]. There are two possibilities: either $r(t)$ is bounded; in which case we have, for all $t \geq 0$

$$
0<C_{1} \leq \operatorname{det} d \phi(t) \leq C_{2} .
$$

Thus using (4.44), we deduce that

$$
\int_{S^{2}}|u(t)| d \mu_{0} \leq \text { Cste. }
$$

By the energy inequality (1.14), estimate (4.46) implies

$$
\int_{S^{2}}|\nabla u(t)|^{2} d \mu_{0} \leq \text { Cste. }
$$


So, using Poincaré's inequality (4.4), estimates (4.46) and (4.47) yield

$$
\|u(t)\| \leq \text { Cste . }
$$

Otherwise, there exist a sequence $t_{n} \rightarrow+\infty$ and $P_{\infty} \in S^{2}$ such that $r\left(t_{n}\right) \rightarrow$ $+\infty$ and $P\left(t_{n}\right) \rightarrow P_{\infty}$. From (4.44), there exist a subsequence of $t_{n}$, that we will also call $t_{n}$, and a function $v_{\infty} \in H$, such that

$$
\begin{cases}v\left(t_{n}\right) \rightarrow v_{\infty} & \text { weakly in } \quad H \\ v\left(t_{n}\right) \rightarrow v_{\infty} & \text { strongly in } \quad L^{2}\left(S^{2}\right)\end{cases}
$$

Let $r>0$ and set $A_{n}=\left(\phi\left(t_{n}\right)\right)^{-1}\left(B\left(P_{\infty}, r\right)\right)$; we have

$$
\begin{aligned}
& \left|\int_{S^{2}} f \circ \phi\left(t_{n}\right) e^{2 v\left(t_{n}\right)} d \mu_{0}-\int_{A_{n}} f \circ \phi\left(t_{n}\right) e^{2 v\left(t_{n}\right)} d \mu_{0}\right| \\
& \leq \sup _{x \in S^{2}} f(x)\left(\operatorname{Vol}\left(A_{n}^{c}\right) \int_{S^{2}} e^{\left|4 v\left(t_{n}\right)\right|} d \mu_{0}\right)^{1 / 2} .
\end{aligned}
$$

Since $\lim _{n \rightarrow \infty} \operatorname{Vol}\left(A_{n}\right)=\operatorname{Vol}\left(S^{2}\right)$ and in view of (4.43), we deduce that

$$
\int_{B\left(P_{\infty}, r\right)} f e^{2 u\left(t_{n}\right)} d \mu_{0}=\int_{A_{n}} f \circ \phi\left(t_{n}\right) e^{2 v\left(t_{n}\right)} d \mu_{0}=\int_{S^{2}} f \circ \phi\left(t_{n}\right) e^{2 v\left(t_{n}\right)} d \mu_{0}+\epsilon_{n},
$$

with $\lim _{n \rightarrow \infty} \epsilon_{n}=0$. From equation (4.37), we have

$$
\int_{S^{2}} f \circ \phi\left(t_{n}\right) e^{2 v\left(t_{n}\right)} d \mu_{0}=8 \pi,
$$

thus it follows from (4.48) that

$$
\lim _{n \rightarrow \infty} \int_{B\left(P_{\infty}, r\right)} f e^{2 u\left(t_{n}\right)} d \mu_{0}=8 \pi .
$$

This completes the proof of Lemma 3.

We are now in position to prove Theorem 3. Since $u_{0}$ is $G$-invariant, by using the uniqueness of the solution $u$, it is not difficult to see that $u$ is also $G$-invariant. From the energy inequality (1.14), we may suppose that

$$
J(u(t))<J\left(u\left(t^{\prime}\right)\right) \text { for all } t>t^{\prime},
$$

otherwise, from the uniqueness of the solution $u$ we would have $u(t) \equiv u_{0}$ for all $t \geq 0$.

(i) The case $\Sigma=\emptyset$. Suppose that $r(t)$ is not bounded. Since $\Sigma=\emptyset$, there exists $R \in G$ satisfying $R\left(P_{\infty}\right) \neq P_{\infty}$. Now, as $\lim _{n \rightarrow \infty} \phi\left(t_{n}\right)(x)=P_{\infty}$ 
a.e., it follows that for $r$ sufficiently small, $\phi\left(t_{n}\right)(x) \notin B\left(R\left(P_{\infty}\right), r\right)$ when $n$ is large enough. Thus

$$
\lim _{n \rightarrow \infty} 1_{\left(\phi\left(t_{n}\right)\right)^{-1}\left(B\left(R\left(P_{\infty}\right), r\right)\right)}(x)=0
$$

where $1_{A}$ denotes the characteristic function of a set $A$. Moreover, we can also choose $r$ such that $B\left(R\left(P_{\infty}\right), r\right) \cap B\left(P_{\infty}, r\right)=\emptyset$. Using (4.43) and (4.50), we have

$$
\lim _{n \rightarrow \infty} \int_{\left(\phi\left(t_{n}\right)\right)^{-1}\left(B\left(R\left(P_{\infty}\right), r\right)\right)} f \circ \phi\left(t_{n}\right) e^{2 v\left(t_{n}\right)} d \mu_{0}=0 .
$$

Since $u$ and $f$ are $G$-invariant and by (4.51), we obtain

$$
\lim _{n \rightarrow \infty} \int_{B\left(P_{\infty}, r\right)} f e^{2 u\left(t_{n}\right)} d \mu_{0}=\lim _{n \rightarrow \infty} \int_{\left(\phi\left(t_{n}\right)\right)^{-1}\left(B\left(R\left(P_{\infty}\right), r\right)\right)} f \circ \phi\left(t_{n}\right) e^{2 v\left(t_{n}\right)} d \mu_{0}=0 .
$$

This contradicts estimate (4.45) in Lemma 3, thus $u$ is uniformly bounded in $H$.

(ii) The case $\Sigma \neq \emptyset$. Suppose that $r(t)$ is not bounded. If $P_{\infty} \notin \Sigma$, then as in case (i) above, we arrive at a contradiction. Otherwise by letting $n \rightarrow \infty$ in (4.37) (with $v_{P, r}=v\left(t_{n}\right)$ and $\phi_{P, r}=\phi\left(t_{n}\right)$ ), we have

$$
f\left(P_{\infty}\right) \int_{S^{2}} e^{2 v_{\infty}} d \mu_{0}=8 \pi
$$

and a computation shows that

$$
\lim _{n \rightarrow \infty} \int_{S^{2}} e^{2 u\left(t_{n}\right)} d \mu_{0}=\lim _{n \rightarrow \infty} \int_{S^{2}} e^{2 v\left(t_{n}\right)} d \mu_{0}=\int_{S^{2}} e^{2 v_{\infty}} d \mu_{0} .
$$

By Onofri's inequality (see [20]) and (1.14), we obtain for large $n$

$$
\frac{1}{4 \pi} \int_{S^{2}} e^{2 u\left(t_{n}\right)} d \mu_{0} \leq e^{J\left(u\left(t_{n}\right)\right) / 4 \pi} \leq e^{J(u(1)) / 4 \pi} .
$$

Combining (4.52), (4.53) and (4.54), we deduce that

$$
2 \leq f\left(P_{\infty}\right) e^{J(u(1)) / 4 \pi} .
$$

Since we have from (4.49), $J(u(1))<J\left(u_{0}\right)$, it follows that

$$
f\left(P_{\infty}\right)>2 e^{-J\left(u_{0}\right) / 4 \pi}
$$

thus contradicting hypothesis (1.16) of Theorem 3. This completes the proof of Part (ii) of Theorem 3.

Proof of Corollary 2. Let $f$ satisfy estimate (1.17). If $\int_{S^{2}} f \circ \phi_{P_{0}, r_{0}} d \mu_{0} \leq$ 0 , then $\sup _{P \in \Sigma} f(P) \leq 0$, so condition (1.16) in Theorem 3 is satisfied for any $G$-invariant data $u_{0} \in X$. On the other hand, if $\int_{S^{2}} f \circ \phi_{P_{0}, r_{0}} d \mu_{0}>0$, we 
let $u^{*}=\frac{1}{2} \log \left(\operatorname{det} d \phi_{P_{0}, r_{0}}^{-1}\right)$. A computation shows that $J\left(u^{*}\right)=0$. Now set $u_{0}=u^{*}+C$, where $C$ is a constant satisfying

$$
e^{2 C} \int_{S^{2}} f \circ \phi_{P_{0}, r_{0}} d \mu_{0}=8 \pi
$$

From (4.55), we see that $u_{0} \in X$. Since $P_{0} \in \Sigma$, it is easy to check that $u_{0}$ is $G$-invariant. We see therefore that condition (1.16) of Theorem 3 is equivalent to

$$
\sup _{P \in \Sigma} f(P) \leq \frac{1}{4 \pi} \int_{S^{2}} f \circ \phi_{P_{0}, r_{0}} d \mu_{0}
$$

this completes the proof of Corollary 2.

\section{REFERENCES}

[1] T. Aubin, Equations différentielles non linéaires et problème de Yamabe concernant la courbure scalaire, J.Math.Pures Appl. 55 (1976), 269-296.

[2] T. Aubin, Meilleures constantes dans le théorème d'inclusion de Sobolev et un théorème de Fredholm non linéaire pour la transformation conforme de la courbure scalaire prescrite, J. Funct. Anal. 32 (1979), 148-174.

[3] T. Aubin, Sur le problème de la courbure scalaire prescrite, Bull. Sci. Math. (5) 118 (1994), 465-474.

[4] J. Bartz - M. Struwe - R. Ye, A new approach to the Ricci flow on $S^{2}$, Ann. Scuola Norm. Sup. Pisa Cl. Sci. (4) 21 (1994), 475-482.

[5] S. Bismuth, Prescribed scalar curvature on a $C^{\infty}$ compact Riemannian manifold of dimension two, Bull. Sci. Math. 124 (2000), 239-248.

[6] S. Y. A. Chang - P. C. YAng, Prescribing Gaussian curvature on $S^{2}$, Acta Math. 159 (1987), 215-259.

[7] S. Y. A. ChAng - P. C. YAng, Conformal deformations of metric on $S^{2}$, J. Differential Geom. 27 (1988), 259-296.

[8] S. Y. A. ChANG - M. J. GuRSKy - P. C. YANG, The scalar curvature equation on 2- and 3-spheres, Calc. Var. Partial Differerential Equations 1 (1993), 205-229.

[9] W. Chen - C. Li, A note on Kazdan-Warner type conditions, J. Differential Geom. 41 (1995), 259-268.

[10] W. CHEN - C. Li, A necessary and sufficient condition for the Nirenberg Problem, Comm. Pure Appl. Math. 47 (1995), 657-667.

[11] W. Chen - W. X. Ding, Scalar curvature on $S^{2}$, Trans. Amer. Math. Soc. 303 (1987), 365-382.

[12] X. X. Chen, Calabi flows in Riemannian surfaces revisited; a new point of view, Int. Math. Res. Not. 6 (2001), 275-297.

[13] B. Chow, The Ricci-Hamilton flow on the 2-sphere, J. Differential Geom. 24 (1986), 153-179.

[14] R. Hamilton, The Ricci flow on surfaces, Contemp. Math. 71 (1988), 237-262. 
[15] M. A. Jendoubi, A simple unified approach to some convergence Theorems of L.Simon, J. Funct. Anal. 153 (1998), 187-202.

[16] J. KAZDAN - F. WARNER, Curvature functions for compact 2-manifolds, Ann. of Math. 99 (1974), 14-47.

[17] J. KAZDAN - F. WARNER, Existence and conformal deformation of metrics with prescribed Gaussian and scalar curvatures, Ann. of Math. 101 (1975), 317-331.

[18] J. Moser, A sharp form of an inequality by N. Trudinger, Indiana Univ. Math. J. 20 (1971), 1077-1092.

[19] J. Moser, On a nonlinear problem in differential geometry, In: "Dynamical systems" (M. Peixoto eds.), Academic Press, 1973.

[20] E. ONOFRI, On the positivity of the effective action in a theory of random surfaces, Comm. Math. Physics 86 (1982), 321-326.

[21] L. Simon, Asymptotics for a class of non-linear evolution equations, with applications to geometric problems, Ann. of Math. 118 (1983), 525-571.

[22] M. Struwe, Curvature flows on surfaces, Ann. Scuola Norm. Sup. Pisa (5) 1 (2002), 247-274.

[23] X. XU - P. YAng, Remarks on prescribing Gauss Curvature, Trans. Amer. Math. Soc. 336 (1993), 831-840.

Département de Mathématiques Université de Brest 6, Avenue Le Gorgeu 29285 Brest, France Ali.Fardoun@univ-brest.fr Paul.Baird@univ-brest.fr Rachid.Regbaoui@univ-brest.fr 